\title{
Applications of Human Biometrics in Digital Image Processing
}

\author{
Anshul Kumar Singh1, Brajesh Kumar Singh2 \\ Department of C. S. E. \\ R. B. S. Engineering Technical Campus, Agra, U.P., 283105, India12
}

\begin{abstract}
Digital image processing is the trending topic of research in recent time and big amount of research work related to Biometric features is done and currently it achieved good amount of accuracy. Biometric features is used for security, verification and recognition purpose. This paper is a showcase of how security systems can be developed by using biometric features of human like face, fingerprint and iris, etc. It can be used for the purpose of identification, recognition and Authentication and it is also applicable for making software for image preparation in bioscience laboratories that make use of scanned or digitally photographed images. The widespread use of such image processing techniques using photography and microscope imaging across the natural science with particular attention being paid to research in cell and molecular bioscience. This paper is a review of various methods trending to the field of biometric applications on biotechnologies.
\end{abstract}

Keywords: - Digital Image Processing, Biotechnology, Bioscience, Biometric.

\section{INTRODUCTION}

Digital image processing is used in the field of Biometric, Biotechnology and Bioscience for past several decades. Biotechnology is the use of living systems and organisms to develop or make any product or any technological application that uses biological systems. Author used biometric features of human or living organisms, and various author uses biometric features such as face, fingerprint, iris, and palm for the purpose of identification, recognition and authentication. And on the other hand digital image processing also have applications in the field of Bioscience. For example study of cell and molecules where author requires scanned or digitally photographed images which can be obtained through microscope and various operations can be performed by using digital image processing software's to study about cells and molecules. We have presented a review of various works done by author in the field of biometrics, biotechnology and bioscience. We tried to put a relation between digital image processing and biotechnology or bioscience. Unique biometric extracted features of human are used in security systems to identify a person; fingerprint is mainly used biometric feature that can be used to recognize a particular person and many other biometric features of a person can be used for security, surveillance, collage campus, government organizations, and in military etc., video based face recognition is used for real time surveillance which helps to keep track of a person. It can be useful in various civil and defense places.

\section{RELATED WORK}

\section{$>$ Finger Print}

Fingerprint is one of the most talked about biometric feature and have been used by various authors. Every person's fingerprint is unique and have been an invaluable feature for law enforcement and forensics science, fingerprint based identification or recognition is the mostly used and reliable biometric feature for automated person identification and recognition, its uniqueness and un-changeability over a period of time made it the most trusted tool that is widely used by many police agencies. The fingerprint recognition is a very long process if the stored one are in millions. With the help of Classification, recognition take less time. Automated fingerprint recognition is the best approach to identify individual. 
E. Marasco et al. [1] discusses various issues related to the vulnerability of fingerprint recognition systems to attack the system with artificial fingerprints, where materials such as, silicone, playdoh, and gelatin are inscribed with fingerprint ridges. Researchers have experimented that some fingerprint recognition systems can be deceived/attacked when these artificial fingers are placed on the sensor, the system successfully allowed false/artificial fingerprints. Various anti spoofing schemes are announced to overcome this issue that are hardware-based, several software-based approaches.

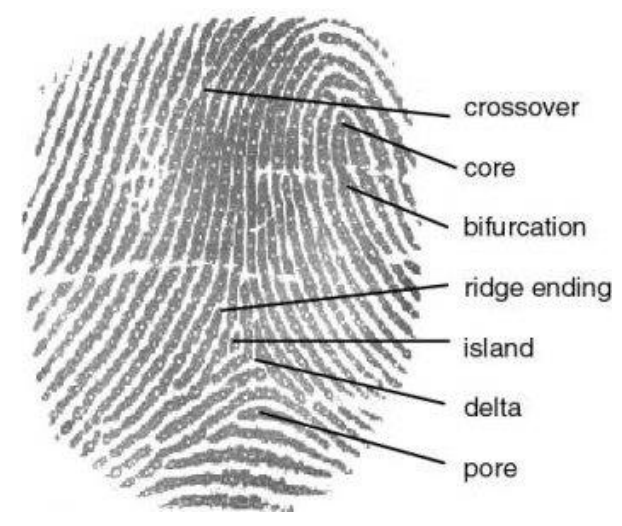

Fig 1:- Finger Print

\section{Face Recognition}

S. A. Patil et al. [2] discusses face recognition is a visual pattern recognition problem as face has factors affecting illumination, pose, expressions. It is converted to two-dimensional image for the purpose of identification. In face recognition system test image is localized and normalized under pre-processing steps and generally consists of four steps: Detection, Alignment, Feature extraction, and Matching. In video based face recognition, the detected faces need to be tracked using a face tracking components. Accuracy is achieved by Face alignment. Facial components, such as eyes, nose, and mouth and facial outline, are located; based on the location points, geometrical transform or image morphing is used to normalize face image with respect to geometrical properties, such as size and pose. Face image is need to be normalized with respect to illumination and gray scale. Geometrically and photo metrically, feature extraction is performed to provide effective information that is useful for distinguishing between faces of different persons and stable with respect to the geometrical and photometrical variations by normalization. The extracted features of the input face is matched against those of enrolled faces in the database or dataset, if it has a desired confidence value then the face is matched otherwise not.

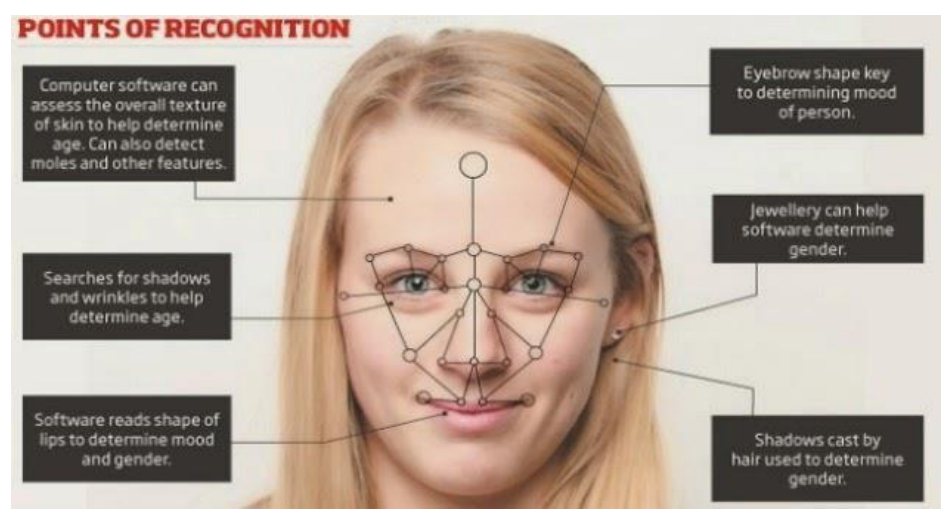

Fig 2:- Face Recognition

Results of face recognition mainly rely on extracted features of face to identify patters and classification methods are used to recognise face.

\section{> Iris Recognition}

J. Daugmen [3] proposed an algorithm to recognize individual by their iris patterns and it turn out to be most unique and reliable biometric feature after fingerprint. Every person have unique iris patterns and it became interesting alternative approach to reliable visual recognition of humans when imaging system is placed at distance less than a meter, and it works fast with very large databases without any false match even with database size in millions. The iris has the great mathematical advantage that its pattern variability among different persons is enormous. It is internal yet fully visible and well protected feature and it does not change over a period of time.

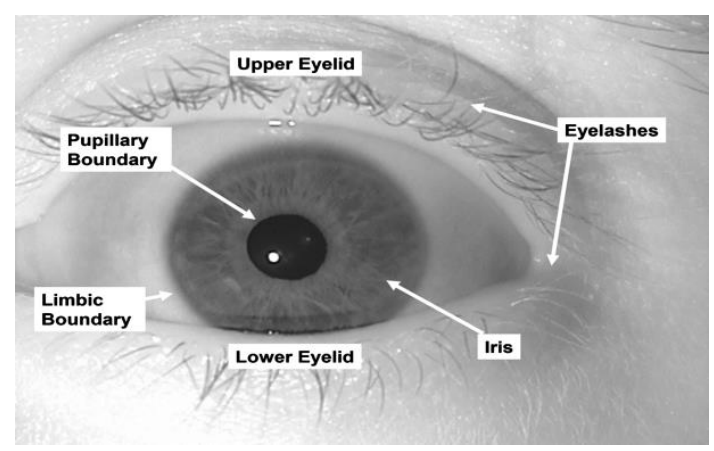

Fig 3:- Iris Recognition 


\section{Study of Cells and Molecules}

M. H. Sarrafzadeh et al. [4] proposed image processing based on Red Green and Blue color analysis to analyse cell concentration of three microalgae i.e. Botryococcus, baraunii, Chlorella vulgaris and Ettila. The experiment were made using concentrated and diluted cultures of microalgae to prepare different concentrations of dry cell weight. Charge Couple Camera is used to image these samples. This method is simple but efficient technique for microalgae biomass estimation. This method is very useful and can be a base for biomass measurement.

\section{> Biometric Security System for surveillance}

A.Singh et al [5] proposed biometric security system to identify miscreants in a real time scenario. It is generally a real time surveillance system which can be placed anywhere to ensure security in mobile area where people are roaming. Principal Component Analysis (PCA) is used with Nearest Neighbour classification. Threshold value is replaced by confidence value. If the test image is found to be matched with the database of stored images with desired confidence value then it will set an alarm and recognize the individual/miscreant.

\section{LITERATURE REVIEW}

Yang et al. [1] worked on well known traditional face recognition approach called Principal Component Analysis (PCA) and they measured the performance of PCA in all directions and they analyse using PCA with high dimensional images takes more time to process and to calculate covariance matrix. Fortunately, they don't need to calculate covariance matric because Eigenvectors can also be calculated by Singular Value Decomposition (SVD) method. But by using SVD, efficiency is very less, transformation from 2D to 1D metrics is not needed. This approach is fully based on 2D metrics as it is more efficient and time saving in comparison to 1D approach.

Gottumukkal et al. [2] gave modular approach PCA, which works in partition of image. Face image is divided in various module. PCA is applied on each part and this method provide more accurate and fast results when compared to conventional PCA under condition of large variations in brightness, facial expressions and illuminations. One limitation is that, it does not work well under the condition of pose variance

Parsi et al. [3] proposed an approach where they matches individual present on a watchlist and they have used their own classifier for the recognition purpose. Author proposed image morphing technique where source image is matched to destination image and gray level of the image is matched. Author used Active Shape Model (ASM) to locate feature points in a face image.

Lee et al. [4] proposed an approach to recognize individual form video sequence. In this method, low dimensional manifold Images are used to enrol users. It is a big challenge to recognize individual with having different variations to the pose. Hausdorff distance is used between the image and its manifold. Temporal information can be easily approximated with image having high variation. Transition matrix is used for storing temporal information which can be helpful to recognize any person face with partial occlusion. Limitation which author found is that careful alignment need to be done and images are very sensitive to illumination.

Fan et al. [5] proposed methodology for face recognition method by using both spatial and temporal information simultaneously. One big problem which author found in this approach is to properly combine facial and temporal dynamics. Video frame is used to learn view specific manifold it is performed using Locally Linear Embedding (LLE). $\mathrm{K}$ means clustering with LLE is used for some view specific manifold.

\section{APPLICATIONS OF DIP IN BIOMETRIC SYSTEMS}

\section{$>$ Cyber Authentication}

In cyber world, biometric feature of humans help in authentication of user that led to preserving the security of any system. In today era of technology authenticity and security is the major concern. Hence fingerprints are a major key for developing an efficient system. 


\section{Detecting liveliness of human}

The major challenging issue nowadays is to find out that whether any biometric feature belongs to a live person or spoofed one. In that scenario biometric system plays a vital role in detecting the fingerprints, face, and iris which in turns provide security and efficiency to the system.

\section{Law Enforcement Agencies:}

Biometric security system is also used by several law related agencies to identify the culprits in any crime cases. Fingerprints in this field are useful from several years and act as an efficient method.

\section{$>$ In Organizations and Corporate:}

This method is widely used nowadays in several organizations at entry level to identify the real person who has legally the permission to enter in any particular firm.

\section{> In Retail and Grocery shops:}

Biometric systems can also be used in retail and grocery shops e.g. Fingerprints are scanned in several shops at entry level in order to identify the real customer which is helpful for the owner. The scanning of fingerprints of human is done at entry level to identify the actual customer.

\section{Banking Application:}

Biometric security system is helpful in ATM's services also for authentication of user. Security is a challenging goal that needs to be achieved in that case.

\section{> In Colleges and Schools:}

Fingerprint, face and iris recognition have become an efficient technique for attendance purpose of faculty members. The absence and presence of staff members can be find out using these systems. On the other hand, it is also helpful in identifying the actual member of any organization.

\section{v. CONCLUSION}

This paper is a showcase of various novel approaches, methods, and technologies used in the field of image processing with various unique features such as fingerprint, face, and iris for the purpose of recognition. Although these approaches can be combined with other approaches and classification methods. Approaches like PCA, nearest neighbour, $\mathrm{K}$ means clustering, LLE, and morphology can be used to provide better accuracy. Study of molecules and cells in biotechnology is possible with the help of Image Processing. In addition Digital image processing is also applicable in the field of bioscience (Study of cells and molecules) with the help of digital image processing tools.

\section{REFERENCES}

[1]. A.K.Singh, and C. Bhatnagar. Biometric Security System for Watchlist Surveillance. IEEE Trans. Elsevier Procedia Computer Science 46 (2015) 596 603.

[2]. M.H.Sarrafzadeh, H. J. La, and J.Y. Lee. Microalgae biomass quanification by digital image processing and RGB color analysis. Springer Science + Business Media. $24^{\text {th }}$ March 2014.

[3]. J. Daugman. How iris recognition works. University of Cambridge, The Computer Laboratory, Cambridge, U.K. 2004

[4]. S. A. Patil and P. J. Deore. Video-based face recognition: a survey. Conference on Advances in Communication and Computing (NCACC'12). April 21, 2012.

[5]. Vikram Panchal. Review on Finger Print Recognition Systems. International

[6]. Journal of Emerging Technologies in Computational and Applied Sciences (IJETCAS), 2013. 